\title{
Optimiser le potentiel d'une communauté de pratique auprès de professionnels en santé : des clés de succès
}

\section{Optimizing the potential of a community of practice with healthcare professionals: keys to success}

\author{
Isabelle SAVARD ${ }^{1, *}$ et Luc CÔTÉ ${ }^{2}$ \\ ${ }^{1}$ Département d'éducation, Université TÉLUQ, Québec, Canada \\ 2 Département de médecine familiale et de médecine d'urgence, Université Laval, Québec, Canada
}

Manuscrit reçu le 9 avril 2020 ; commentaires éditoriaux formulés aux auteurs le 10 décembre 2020 et le 9 janvier 2021 ; accepté pour publication le 13 janvier 2021

\begin{abstract}
Résumé-Contexte et problématique : La «communauté de pratique 》 $(\mathrm{CoP})$ auprès de professionnels en exercice suscite de plus en plus d'intérêt en éducation des sciences de la santé. En tant que stratégie d'apprentissage collaboratif, elle valorise et soutient l'explicitation des savoirs d'expérience en vue du développement des meilleures pratiques dans des contextes précis. Or, ce concept a évolué et génère souvent de la confusion voire de la controverse, d'autant qu'il y a plusieurs types de CoP qui sont initiées tantôt par ses membres, tantôt par des organisations qui utilisent cette stratégie pour être plus performantes. Pour optimiser le potentiel de la $\mathrm{CoP}$, il importe de comprendre sa signification, en plus de connaître les principes de base et les conditions de succès pour sa planification, son déroulement et son évaluation. Buts : Résumer le concept évolutif de la CoP, dégager ses principes de base et décrire les principales conditions de succès pour sa planification, son déroulement et son évaluation. Méthodes et résultats : En se référant à la littérature et aux expériences d'animation de CoP d'un des auteurs, ceux-ci en résument le concept évolutif et les principaux principes, puis décrivent sept clés de succès en y associant les principales actions à prévoir pour chacune d'elles. Conclusions : La CoP s'avère être une stratégie d'apprentissage collaboratif des plus pertinentes pour les enseignants en sciences de la santé et leurs organisations qui veulent améliorer les pratiques. Il est souhaitable que ce guide informatif soit utilisé pour faciliter la tenue de projets de CoP et pour en évaluer les impacts.
\end{abstract}

Mots clés : Communauté de pratique, savoirs d'expérience, clés de succès, professionnels de la santé

\begin{abstract}
Context and issues: There is a growing interest in "community of practice" (CoP) among practising professionals in health science education. As a collaborative learning strategy, it values and supports the elucidation of experiential knowledge for the development of best practices in specific contexts. However, this concept has evolved and is often confusing and even controversial, especially since there are several types of CoPs that are sometimes initiated by its members or by organizations that use this strategy to improve their performance. To optimize the potential of $\mathrm{CoPs}$, it is important to understand their meaning, as well as the basic principles and conditions for success in planning, conducting and evaluating them. Aims: To summarize the evolving concept of $\mathrm{CoP}$, identify its basic principles and describe the main conditions of success for its planning, implementation and evaluation. Methods and results: Referring to the literature and to the CoP facilitation experiences of one of the authors, the authors summarize the evolving concept and the main principles and describe seven keys to success, with the main actions to be planned for each of them. Conclusions: CoP has proven to be a highly relevant collaborative learning strategy for health science educators and their organizations that want to improve practices. It is desirable that this informative guide be used to facilitate the implementation of CoP projects and to evaluate their impacts.
\end{abstract}

Keywords: Community of practice, knowledge from experience, keys to success, health professionals

\footnotetext{
${ }^{*}$ Correspondance et offprints : Isabelle SAVARD. Département d'Éducation, Université TÉLUQ, 455 rue Du Parvis G1K-9H6 Québec, Canada. Mailto: isabelle.savard@teluq.ca.
} 


\section{Contexte et problématique}

Le virage vers des approches visant le développement des compétences a amené les responsables de programmes de formation initiale, avancée et continue à repenser l'offre selon un continuum qui valorise l'apprentissage à vie. Dans ce contexte, les activités de formation professionnelle continue devraient viser l'appropriation de nouveaux savoirs dans une perspective de développement des compétences. Toutefois, différents auteurs ont démontré que les programmes de formation professionnelle continue sont souvent trop courts pour pouvoir prétendre mener au développement de compétences [1-3]. Ils sont également souvent trop détachés de la pratique, du travail quotidien [3]. Il importe alors de trouver des modalités qui permettent de faire le pont entre la formation et le transfert dans la pratique. La communauté de pratique $(\mathrm{CoP})$ constitue une solution intéressante.

La CoP suscite de plus en plus d'intérêt en éducation, en particulier en éducation des sciences de la santé [4-9]. Elle s'avère être une modalité d'apprentissage collaboratif pertinente pour des professionnels en exercice, qui partagent une pratique commune et qui veulent la développer. Or, depuis ses débuts, le concept a beaucoup évolué. Il est interprété de plusieurs façons et donne lieu à divers types de $\mathrm{CoP}$, ce qui contribue aux difficultés à décrire la $\mathrm{CoP}$, son fonctionnement et à en évaluer ses impacts [5,10].

Quelques publications ont fait état des défis liés à l'implantation et au déroulement de la CoP. Par exemple, comment faire en sorte que les membres soient autonomes dans leurs apprentissages si la CoP est imposée par une organisation au lieu d'être initiée par eux? De même, comment susciter et maintenir la motivation des membres à y participer, surtout s'il s'agit de professionnels en exercice qui ont peu de temps pour le faire? Ces publications ont également permis d'identifier des facteurs de succès pour son implantation, son déroulement et son évaluation [11-15].

\section{Objectif}

En nous inspirant de la littérature, des expériences d'un des auteurs (LC) dans l'animation de diverses CoP en éducation des sciences de la santé $[16,17]$ et de ses échanges avec Étienne Wenger, figure de proue dans l'évolution du concept, nous clarifions d'abord ce concept et résumons son évolution, puis détaillons les principales clés de succès en gardant en tête qu'une conception pédagogique soigneusement élaborée est essentielle à l'efficacité d'une CoP [12]. Nous présentons chacune des clés en la décrivant brièvement et en y associant les principales actions à prévoir. Des exemples sont aussi fournis. Nous souhaitons que cet article contribue à enrichir la réflexion des professionnels en exercice et des gestionnaires des milieux de santé intéressés par l'apprentissage collaboratif visant le développement et l'application des meilleures pratiques, notamment en éducation des sciences de la santé.

\section{La communauté de pratique : un concept polysémique}

C'est dans la foulée des théories socio-cognitives [18] et de la théorie de l'apprentissage en contexte - situated learning - [19] que le concept de CoP a été initialement proposé en 1991 par Lave et Wenger [20]. Ces deux auteurs s'intéressaient aux processus d'insertion et de socialisation professionnelles d'un novice (par exemple, un étudiant en début de stage) dans une «communauté» ou milieu de pratique (par exemple, un département clinique hospitalier) dans lequel se côtoient des novices et des personnes plus expérimentées, parmi lesquelles des experts du domaine. Leurs travaux ont montré que les novices migrent d'une participation périphérique attendue - legitimate peripheral participation- à une participation beaucoup plus active au contact des membres de cette «communauté» ayant une culture et des règles de fonctionnement qui lui sont propres. L'observation de pairs, les échanges informels et formels entre les membres, principalement au niveau de leurs pratiques communes, figurent parmi les stratégies d'apprentissage importantes. Ainsi, au cours de ce processus d'intégration où ils jouent un rôle actif, ils deviennent membres à part entière de cette «communauté».

La définition la plus répandue de la CoP est celle de Wenger [21]. Il s'agit d'un groupe de personnes ayant un intérêt partagé et une pratique commune (par exemple, la supervision clinique en sciences de la santé). Les membres se rencontrent périodiquement pour partager leurs expériences, incluant les problématiques vécues sur le terrain, leurs questions, les «trucs » du métier, etc. Par ce partage, ils enrichissent leur répertoire d'actions et contribuent ainsi à faire avancer leur domaine en mettant en valeur leurs savoirs d'expérience. L'engagement mutuel (intérêt et implication des membres à apprendre les uns des autres et à co-construire leurs savoirs), l'entreprise commune (objectifs similaires et explicites visés par les membres) et le répertoire partagé (savoirs formels et savoirs d'expérience, valeurs et routines adoptées par les membres) caractérisent la CoP. Ainsi définie, cette notion est alors devenue un nouveau cadre conceptuel de l'apprentissage et une pratique recommandée dans le domaine du développement pédagogique en sciences de la santé [22].

La dimension socio-constructiviste de la connaissance, qui rend compte des interactions soutenues entre des personnes qui co-construisent et apprennent les unes des autres, et la mise en valeur des savoirs d'expérience des membres sont au cœur de la CoP. C'est ce qui la distingue, par exemple, d'un groupe de travail dans lequel un mandat précis provient habituellement d'une instance extérieure au groupe, telle une direction d'établissement, avec commande d'une production dans un temps défini. Notons que nous assistons ces dernières années à l'émergence de différentes modalités de CoP, en termes de site (membres provenant d'un seul ou de plusieurs milieux), d'ampleur (une dizaine de membres à des centaines), de forme (en 
présence, virtuelle ou hybride) et de durée (de quelques mois à quelques années). De plus, certaines CoP sont initiées par leurs membres, alors que d'autres le sont par des organisations ou institutions. Enfin, certaines CoP fonctionnent en réseau fermé (il n'y a pas de nouveaux membres qui sont admis dans la $\mathrm{CoP}$ une fois celle-ci démarrée) et d'autres en réseau ouvert (de nouveaux membres peuvent se joindre au groupe existant).

À partir de 2002, le centre notionnel du concept a changé, passant d'une manière de comprendre l'apprentissage en contexte de pratique professionnelle à celui d'outil de gestion des connaissances et des problèmes dans un milieu ou une organisation, afin de maximiser son rendement et sa compétitivité $[12,23]$. Ainsi, les milieux des affaires et de la gestion se sont largement approprié cette stratégie pour répondre à leurs besoins organisationnels, liés par exemple à la résolution des problèmes, au développement et à l'implantation des innovations, à la facilitation du transfert des meilleures pratiques.

Cette «migration conceptuelle» a fait l'objet de diverses critiques $[5,10,24]$, notamment celle dénonçant une confusion entre «savoirs dans l'action » et $\mathrm{CoP}$, ou de mises en garde, par exemple à l'égard de la menace de l'autonomie des membres d'une CoP qui serait initiée par une organisation, qui est un enjeu majeur. Récemment, McGrath et al. [5] ont invité à la prudence au sujet de la signification d'une CoP. Leur analyse critique de l'utilisation de ce concept en éducation, incluant son usage dans le champ de la recherche en éducation médicale, a montré que de nombreux articles parmi ceux qu'ils ont analysés n'offraient pas de définition claire et rigoureuse de ce qu'est la CoP. Ils ont conclu que ce concept surutilisé l'est trop souvent d'une manière floue (tout et rien à la fois), ce qui peut conduire à son ambiguïté. De même, Buckley et al. [25] recommandent de clarifier le sens que nous accordons à la $\mathrm{CoP}$ lorsque nous en parlons.

Aussi, nous retenons dans cet article la définition de 1998 de Wenger précédemment mentionnée. Concrètement, en éducation des sciences de la santé, nous nous intéressons plus particulièrement aux $\mathrm{CoP}$ de dimension restreinte (entre 10 et 20 participants) et de durée limitée (environ une année), qui sont volontairement créées par des professionnels en exercice, habituellement en collaboration plus ou moins soutenue avec leur organisation. Ceux-ci veulent répondre à des besoins spécifiques en lien avec leur pratique commune et partager leurs savoirs d'expérience et leur expertise en vue de la développer. Par exemple, une CoP pourrait rassembler des cliniciens enseignants provenant d'un ou de plusieurs départements d'une faculté de médecine qui se regrouperaient pour améliorer leurs habiletés à superviser des stagiaires ou des internes ayant des difficultés quant au développement de leurs compétences.

\section{Principales clés de succès de la communauté de pratique}

Les sept clés de succès que nous détaillons sont complémentaires et forment un tout cohérent. Bien que chacune d'elles soulève des enjeux ou défis singuliers, certains principes de base sont transversaux; ils s'appliquent donc à toutes les clés et doivent guider la conception, la réalisation et l'évaluation de la CoP :

- la CoP vise avant tout l'amélioration des pratiques des membres tout en est influençant la culture du milieu dans laquelle elle prend place $[6,12,14,15]$;

- elle ne se limite pas au partage des connaissances, dont les savoirs d'expérience; elle en génère de nouvelles dans le contexte du développement de l'expertise et de la pratique [14];

- l'implantation d'une CoP ne peut se faire sans considérer certaines variables culturelles pouvant interférer avec ses conditions gagnantes, dont la relation à l'autorité, aux pairs, au temps [26];

- la vitalité de la communauté dépend de sa capacité à générer suffisamment d'enthousiasme, de pertinence et de valeur pour attirer et engager les membres, de même que pour obtenir l'appui de l'organisation dans laquelle elle émerge [12];

- la survie d'une CoP dépend surtout de la pertinence des objectifs visés et de la qualité des interactions qu'elle génère [12] ;

- le fait de rendre ces principes explicites permet de s'y référer comme cadre conceptuel à appliquer avec flexibilité.

\section{Clé 1 : Former un groupe restreint d'exploration du projet d'une communauté de pratique}

Le démarrage d'une CoP est le fruit d'une démarche de réflexion souvent initiée par une ou quelques personnes désirant enrichir leurs pratiques par des échanges réguliers entre pairs. Il arrive aussi que l'organisation soit l'initiatrice du projet. Des auteurs $[13,15,17]$ suggèrent d'abord de former un groupe restreint afin d'explorer le projet de $\mathrm{CoP}$ et de planifier son démarrage: pourquoi une $\mathrm{CoP}$ plutôt qu'une autre modalité? Quelles sont ses visées, sa valeur ajoutée? Qui est susceptible de participer? Selon quelles modalités? Quelles sont les ressources qui seront requises? Etc. Ces initiateurs (praticiens et gestionnaires) devraient être reconnus comme des leaders crédibles dans le(s) milieu(x) où la CoP se développera [27]. Idéalement, un membre expert de la CoP ou de son animation devrait faire partie de ce groupe. Finalement, soulignons que les réflexions du groupe restreint deviendront des propositions à soumettre aux futurs membres de la CoP. Cesderniers prendront les décisions quant à savoir si la CoP démarrera et à quelles conditions.

\section{Principales actions à prévoir :}

- formuler les objectifs de la $\mathrm{CoP}$;

- s'entendre sur le profil des membres potentiels;

- convenir des modalités de publicité/recrutement et d'identification des besoins des futurs membres;

- discuter des modalités de sélection d'un animateur;

- prévoir les ressources (humaines, financières, technologiques) dont la CoP aura besoin; 
- amorcer la planification du processus d'évaluation de la CoP.

\section{Clé 2 : Définir des objectifs explicites, pertinents et réalistes pour les participants}

De Carvalho-Filho et al. [15] se sont intéressés aux clés de succès des CoP dans un contexte d'appui au développement pédagogique des enseignants en sciences de la santé. Ils donnent l'exemple de l'émergence d'une CoP d'enseignants dans un milieu qui aurait constaté le manque de cohérence entre les cibles d'apprentissage et les méthodes d'évaluation de ces apprentissages. Cet exemple illustre le contexte qui nous intéresse, soit une CoP initiée par des professionnels en exercice comme stratégie collective pour répondre à des besoins issus de leur pratique dans le but de l'enrichir.

Il importe, dans un premier temps, d'identifier un problème concret et commun vécu par les personnes d'un même milieu (ou provenant de milieux assez similaires) et les besoins qui en découlent, et de s'en servir pour guider la formulation d'objectifs explicites, pertinents et réalistes. On sait que la motivation des participants à l'engagement et à la persévérance dans une activité repose avant tout sur la pertinence des objectifs visés.

À ce sujet, Brien [28] propose trois facteurs influençant la motivation qui, selon nous, peuvent être utilisés pour guider la formulation d'objectifs pertinents et réalistes pour les participants : l'attitude face à la tâche, l'ampleur de la tâche et l'importance accordée aux buts. Kahlke et al. [29] (p. 230) abondent dans ce sens et citent Cook et Artino [30] pour souligner que «...la motivation à agir dépend de la manière dont le comportement associé croise la compétence perçue d'un individu («puis-je le faire?»), son sentiment d'autonomie («ai-je le contrôle sur le résultat?», la valeur anticipée de la récompense qui en résulte («cela en vaut-il la peine?») et la nature des interactions entre un individu et son contexte social («le contexte me permet-il d'agir?»)»[traduction libre]. La planification de la $\mathrm{CoP}$ devrait donc débuter par l'anticipation des réponses à ces questions. Le concepteur attentionné doit absolument se demander : (1) ce que les membres de la communauté répondraient à ces questions ; (2) en discuter avec eux ; (3) lister ce qui serait susceptible d'influencer l'attitude face à la tâche, l'ampleur de la tâche et l'importance accordée aux buts. Dans le cas de CoP initiées par les membres, il faut aussi établir dès le départ comment l'organisation va reconnaître la légitimité de la $\mathrm{CoP}$ et en valoriser la participation [15]. Cette valorisation va influencer l'attitude face à la tâche ainsi que l'importance accordée aux buts.

Un autre défi est de s'assurer que tous les participants saisissent bien la nature et l'orientation de la CoP dans laquelle ils vont s'impliquer. Un des moyens pour y parvenir est de travailler en étroit partenariat avec eux notamment pour identifier les objectifs visés et analyser la cohérence entre les objectifs de la $\mathrm{CoP}$ et le problème ou le but identifié. Au Canada, le Centre facilitant la recherche et l'innovation dans les organisations (Cefrio) [11] a présenté les qualités d'une bonne thématique d'échanges. Elle doit être: assez large pour interpeller un nombre suffisant de participants; assez circonscrite pour qu'ils puissent arriver à des résultats concrets; assez concrète pour leur permettre d'aborder des problèmes réels. En somme, le sujet doit être significatif et passionnant pour les participants. Dans le même ordre d'idée, De CarvalhoFilho et al. [15] précisent que si les leaders de l'organisation dans laquelle émerge la $\mathrm{CoP}$ reconnaissent sa valeur ajoutée, ils seront plus enclins à mettre en place des mesures pour faciliter son démarrage et son bon déroulement.

\section{Principales actions à prévoir :}

- identifier et définir un problème pratique (ou un but de formation) qui permettra d'améliorer la pratique des membres; le travail d'exploration de la problématique se fera avec des praticiens du ou des milieux visés, en collaboration avec des représentants de ces milieux;

- formuler des objectifs clairs, qui se réfèrent à des comportements observables, car ils devront être évalués. Si la CoP vient en appui à un programme formel de formation (par exemple, programme de développement professionnel continu), les objectifs de la CoP doivent évidemment être en cohérence avec ceux du programme ; - analyser la cohérence entre le problème (ou le but identifié) et les objectifs fixés : est-ce que les objectifs (ou le but) sont suffisamment importants pour maintenir la motivation des participants et aider à solutionner la problématique?;

- lister les éléments qui pourraient influencer l'attitude des participants face à la tâche, à l'ampleur de la tâche et à l'importance accordée aux objectifs (ou au but);

- identifier des possibilités de thèmes d'échanges/activités et de personnes-ressources a priori pertinentes pour la CoP.

\section{Clé 3 : Préciser les règles de fonctionnement et trouver le bon rythme}

Pour favoriser le bon déroulement des rencontres et faciliter l'atteinte des objectifs visés, des règles de fonctionnement et de communication explicites doivent être adoptées dès le départ par les membres, en veillant à les ajuster selon l'évolution des besoins, au regard, par exemple, de la fréquence, la durée, du contenu et des modalités des rencontres, du processus décisionnel, de la confidentialité des échanges, de l'intégration ou non de nouveaux membres dans la $\mathrm{CoP}$, etc. Ces règles doivent être claires et adaptées, sans être trop contraignantes, car trop de contraintes limiteraient le goût de participer et la production de nouvelles idées lors des échanges.

Par ailleurs, comme le soulignent Wenger et al. [12], le rythme de la communauté est le plus fort indicateur de sa vitalité. Il fait référence non seulement à la fréquence et à la durée des rencontres, mais aussi au degré d'intensité de chacune de ces rencontres. Par exemple, certaines 
rencontres peuvent soulever des enjeux particuliers ou des désaccords et susciter des réactions. Il importe de garder un rythme constant, car il n'y a rien de pire que d'annuler une ou des rencontres planifiées. Cela risque d'inciter les participants à douter de la pertinence des rencontres, voire de la CoP, et à planifier d'autres activités en parallèle, ce qui, à moyen terme, nuira considérablement à la motivation, au taux de participation et à la qualité des échanges. Un rythme trop intense risque «d'essouffler » les participants et d'entraîner une diminution de participation, car ils seront débordés. Par contre, si le rythme est trop lent, ils risquent d'avoir l'impression de perdre leur temps et la qualité des réflexions et discussions s'en ressentira. Bien que les contextes de $\mathrm{CoP}$ varient beaucoup, selon notre expérience, des rencontres selon une périodicité de quatre à cinq semaines permettent habituellement de développer et de maintenir la motivation, le sentiment d'appartenance et la productivité. De plus, ce rythme laisse suffisamment de temps pour la réflexion, le transfert des apprentissages dans la pratique et pour l'analyse des effets de ce transfert.

\section{Principales actions à prévoir :}

- déterminer le «lieu» des rencontres: seront-elles en présence, virtuelles (en recourant, par exemple, à : Zoom $^{\circledR}$, WhatsApp ${ }^{\circledR}$, Facetime ${ }^{\circledR}$ ou autres) ou hybrides (certaines rencontres en présence et d'autres à distance); le cas échéant, s'assurer que l'infrastructure et le soutien technologiques requis seront disponibles;

- déterminer si la CoP sera fermée (quelques personnes participent en raison de leur expertise/implication en lien avec la problématique) ou ouverte à tous ceux qui ont un intérêt pour cette problématique (ce qui implique un plus grand nombre de participants) et en analyser les implications ;

- convenir de la fréquence et de la durée approximative des rencontres;

- discuter si les rencontres seront enregistrées ou non, en considérant les avantages (par exemple: accès aux discussions a posteriori) et les inconvénients (certains membres, par exemple, pourraient se sentir menacés et parler moins librement);

- déterminer à quel moment et selon quelles modalités les membres de la CoP recevront des documents en lien avec les rencontres antérieures et à venir, des rappels au sujet des rencontres à venir, des documents en lien avec les rencontres, etc. ;

- déterminer qui animera la $\operatorname{CoP}$ (voir clé 4).

\section{Clé 4: Choisir un animateur et clarifier son rôle}

Certaines CoP fonctionnent en ayant un animateur formel. Dans d'autres, les membres jouent ce rôle tour à tour, alors qu'une autre modalité consiste à ne pas avoir d'animateur attitré. Notre expérience nous indique que la présence d'un animateur formel est nécessaire puisqu'il joue un rôle majeur dans la mise en place et dans le développement de la CoP. Nous recommandons donc qu'il $\mathrm{y}$ en ait un qui soit un praticien comme ses collègues, pour des raisons de crédibilité. Si un animateur est recommandé par le comité de démarrage, les membres de la CoP devront être consultés.

L'animateur s'assure que la CoP évolue selon les objectifs visés tout en maximisant la réflexion et l'échange. Étant un facilitateur du partage de connaissances et d'expériences (satisfaisantes et plus difficiles), l'animateur, en plus de bien communiquer, écoute, questionne, stimule et soutient. Il veille notamment au partage du temps de parole, sans toutefois prendre pour acquis que tous les participants doivent avoir un temps de parole égal. En effet, selon Wenger et al. [12], il faut éviter de chercher à faire participer tous les membres également et respecter le fait qu'il y ait diverses façons de participer; la valorisation de la différence doit donc être recherchée. Finalement, c'est souvent l'animateur qui est le lien entre la CoP et l'organisation dans laquelle elle évolue (par exemple, une CoP composée de cliniciens enseignants d'un département et la direction de ce département ou de la faculté).

Un autre défi important pour l'animateur est de trouver le bon niveau de «directivité ». Veiller à l'atteinte des objectifs est une chose, le faire avec rigidité en est une autre. Roberts [24] et Pemberton et al. [31] soulignent qu'il devrait développer des stratégies pour atténuer la hiérarchie au sein de la CoP, en assurant un dialogue horizontal entre les membres, en encourageant la collaboration et la cocréation plutôt que la concurrence. Ceci est en relation avec une des variables culturelles identifiées par Savard [26]: la relation à l'autorité. En effet, le « dialogue à l'horizontal » pourrait s'avérer beaucoup plus difficile à mettre en pratique pour certains participants ou animateurs, habitués à vivre dans une hiérarchie forte et omniprésente. Bref, l'animateur fait confiance aux membres de la CoP et les aide à faire en sorte que la communauté atteigne son plein potentiel, notamment en rendant les règles de communication et de fonctionnement explicites. Comme le soulignent Wenger et al. [12], «[...] l'évolution est commune à toutes les communautés et le rôle principal de l'animateur est de catalyser cette évolution» (p.3). L'animateur joue certes un rôle clé, mais il doit garder en tête qu'une communauté «ne se dirige pas, elle s'anime» [13] (p. 14) ; il est donc au service de la communauté et non l'inverse.

\section{Principales actions à prévoir :}

- clarifier le rôle de l'animateur (par exemple, préparer un aide-mémoire à l'attention des participants et de l'animateur, auquel ils pourront se référer) ;

- discuter avec les participants des stratégies d'animation que l'animateur pourra utiliser, au besoin ;

- rendre explicites les règles de communication et de fonctionnement ;

- préparer un gabarit pour orienter la structure des synthèses, en cohérence avec les décisions prises ; 
- prévoir que l'animateur tienne un journal de bord pour suivre l'évolution de la $\mathrm{CoP}$;

- prévoir que l'animateur rencontre le représentant de l'organisation et préciser le type d'informations qu'il pourra lui transmettre.

\section{Clé 5 : S'engager et s'impliquer activement comme participant}

La réussite de la $\mathrm{CoP}$ engage la responsabilité de chacun des membres. Les participants développeront le sentiment d'appartenance et s'investiront dans ses activités s'ils perçoivent au fil des rencontres qu'il y a plus d'avantages que d'inconvénients à y participer; il faut donc qu'il y ait perception d'une valeur ajoutée. Tirer des bénéfices personnels est un facteur essentiel de motivation, surtout quand on a à gérer le manque de temps. Pour que la CoP atteigne ses objectifs, il est essentiel que les thématiques des activités soient stimulantes et qu'elles correspondent aux besoins des membres. De même, la réponse à ces besoins doit être valorisée et soutenue par l'organisation dans laquelle se déroule la $\mathrm{CoP}$. Mais par-dessus tout, l'implication active des participants dans les échanges sur leur pratique doit être et rester la pierre angulaire de la vitalité de la CoP. C'est aussi ce qui contribuera à sa crédibilité.

Pour favoriser l'intérêt et la participation, De Carvalho-Filho et al. [15] suggèrent d'inclure parmi les membres des personnes plus expérimentées reconnues pour leur expertise et d'autres reconnues pour leurs idées novatrices. Une variante est d'inviter ce type de personnes à des moments jugés opportuns [15]. Différents auteurs recommandent aussi de dégager du temps pour participer $[11,15,17]:$ «es membres de la CoP doivent réserver du temps pour lire, comprendre, réfléchir et appliquer les nouvelles connaissances aux problèmes et défis...» [traduction libre] [15] (p. 4). Wenger et al. [12] identifient que protéger du temps est particulièrement important dans les premiers stades de développement d'une $\mathrm{CoP}$ car c'est à ce moment que les membres apprennent à se connaître et à travailler ensemble, tout en développant le lien de confiance. Finalement, notons que le fait d'avoir du temps reconnu par l'organisation pour participer, et des incitatifs tels que des attestations de participation, des crédits de formation continue, figurent parmi les facteurs de succès [17].

Principales actions à prévoir :

- clarifier le type de participation attendue;

- inventorier des moyens concrets et réalistes de susciter la confiance et la participation active (par exemple: accès au profil des membres, prévoir une courte activité où les membres se présentent; réflexions et discussions centrées sur la pratique);
- déterminer comment seront mises en valeur les expertises/expériences des participants (planifier des stratégies de mise en valeur que l'animateur pourra utiliser);

- faire valoir la position d'apprentissage individuelle nécessaire à l'efficacité de la $\mathrm{CoP}$ (au besoin, briser les barrières hiérarchiques qui pourraient nuire à la curiosité et à l'ouverture aux idées nouvelles);

- déterminer des stratégies concrètes, tant du côté des participants que du côté de l'institution, pour protéger du temps (avant, pendant et entre les rencontres);

- maintenir les contacts avec les membres entre les rencontres par des moyens faciles à utiliser (par exemple, courriels, site de dépôt de documents).

\section{Clé 6 : Mettre les « savoirs d'expérience » au cœur des échanges}

Comme mentionné précédemment, une des spécificités de la $\mathrm{CoP}$ est que les membres apprennent les uns des autres, à partir de leurs expériences respectives. C'est essentiellement ce partage issu de la pratique (répertoire expérientiel partagé) qui différencie la $\mathrm{CoP}$ d'autres formes d'apprentissage et qui lui donne sa raison d'être. Un premier défi consiste à mettre ces savoirs d'expérience au cœur des échanges sans pour autant minimiser l'importance des savoirs théoriques ou plus formels. À ce sujet, l'étude de Meagher-Stewart et al. [32] a montré que les participants à une $\mathrm{CoP}$ préféraient discuter avec leurs pairs des savoirs d'expérience, bien qu'ils reconnaissaient l'importance d'autres savoirs comme les données probantes: ils percevaient que les échanges de savoirs d'expérience étaient non seulement utiles pour leur pratique, car ils étaient contextualisés, mais qu'ils contribuaient au climat de confiance et facilitaient la participation (voir clé précédente).

Puisque les savoirs tacites sont souvent intuitifs et implicites, un des moyens de les expliciter est de s'en parler entre participants. À cet égard, l'animateur contribuera à leur explicitation, par exemple en demandant aux membres d'expliquer leur démarche dans telle situation, de parler de leurs bonnes et moins bonnes initiatives en lien avec la problématique discutée, de comparer les stratégies utilisées sur le terrain par les membres, etc. Par leur ouverture et leur bienveillance, ceux-ci s'aideront à minimiser la compétition et la crainte de l'évaluation par les pairs, en particulier lorsque qu'un membre fait état de difficultés dans l'exercice de sa pratique. C'est ainsi que le climat de confiance se développera et que les membres apprendront les uns avec les autres et les uns des autres.

Principales actions à prévoir :

- planifier une activité avec les membres pour définir les types de savoirs et reconnaître la richesse des savoirs d'expérience partagés ; solliciter des exemples auprès des membres ; 
- identifier les sources potentielles de craintes à partager ses savoirs d'expérience;

- prévoir du temps pour aider les membres à réfléchir à des aspects particuliers de leur pratique;

- dresser un inventaire des stratégies évoquées par les membres lors des échanges ;

- prévoir de courts exercices d'appropriation à mettre en pratique d'ici la prochaine rencontre.

\section{Clé 7 : Évaluer la communauté de pratique en continu}

En lien avec la clé 1, nous avons suggéré que les membres du groupe restreint d'exploration/démarrage amorcent la planification du processus d'évaluation de la CoP. Idéalement, nous suggérons que l'évaluation se fasse en continu car elle permettra d'apporter les modifications jugées nécessaires au fur et à mesure du déroulement de la CoP. Ceci contribuera à la motivation et à la satisfaction des membres. On peut, par exemple, recueillir auprès des membres quelques informations à la fin de chaque rencontre (ce qui a bien et moins bien fonctionné pendant la rencontre, les questions qui restent en suspens) et faire une évaluation plus complète de la $\mathrm{CoP}$ à la fin.

L'évaluation devrait globalement porter autant sur le contenu (par exemple, objectifs atteints ou pas, apprentissages réalisés ou pas et pourquoi, aspects organisationnels, principaux points positifs, points à améliorer, etc.) que sur le processus de participation, (par exemple, vitalité des échanges, satisfaction des membres). Le recueil des données sera d'autant plus riche si on utilise une combinaison des méthodes (par exemple, entrevue de groupe et questionnaire individuel), si le recueil permet de générer des données qualitatives et quantitatives et si divers acteurs-clés participent à la démarche : membres du groupe de démarrage, membres de la $\mathrm{CoP}$, incluant l'animateur, représentant de l'organisation et autres personnes ayant joué un rôle significatif.

Côté et al. ont utilisé un devis de recherche-action auprès de deux $\mathrm{CoP}$ de cliniciens enseignants en sciences de la santé $[16,17]$. Il s'agit d'une approche de recherche qualitative, de nature participative, où les chercheurs et les praticiens-acteurs collaborent étroitement afin de résoudre une ou des situations problématiques en contexte réel, et de générer des connaissances; les connaissances et l'action s'influencent mutuellement. La démarche s'articule autour d'une série d'activités (celles de la $\mathrm{CoP}$ ) qui constituent des sources de collecte et d'analyse des données. Les activités se structurent et se raffinent à partir de cycles d'exploration-action-réflexion-évaluation, ce qui permet notamment d'impliquer les membres de la $\mathrm{CoP}$ dans l'évaluation en continu, d'évaluer les retombées de la $\mathrm{CoP} a ̀$ divers moments de son déroulement et de les ajuster au besoin. Nous recommandons aussi de relancer les participants quelques mois après que la $\mathrm{CoP}$ ait pris fin afin d'évaluer si les changements de pratique ont des effets durables et de dégager des pistes pour y parvenir, le cas échéant (Encadrés 1 et 2).

Principales actions à prévoir :

- déterminer qui pilotera l'évaluation;

- préciser sur quoi l'évaluation portera et comment elle sera réalisée;

- prévoir des mécanismes d'évaluation continue;

- déterminer comment les données issues de l'évaluation seront utilisées, analysées et diffusées.

\section{Conclusion}

La CoP s'avère être une stratégie des plus pertinentes pour les praticiens et leurs organisations. Elle favorise l'apprentissage collectif en valorisant le partage des savoirs d'expérience et leur intégration aux savoirs plus formels, ce qui contribue au développement de l'expertise. Mais elle n'est pas une panacée et comporte des défis qu'il convient d'anticiper en mettant en place des conditions facilitantes pour sa mise en route et son développement. Nous espérons que les principes et les clés de succès présentés seront utiles et qu'ils aideront à faire avancer les pratiques et les recherches dans ce domaine d'intérêt en sciences de la santé.

\section{Contributions}

Les deux auteurs ont collégialement travaillé à la rédaction des diverses versions de cet article.

\section{Liens d'intérêt}

Aucun auteur ne déclare de conflit d'intérêt en lien avec le contenu de cet article.

\section{Approbation éthique}

Non requise. 


\section{Encadré 1. Deux exemples d'expériences de communauté de pratiques réussies}

Nous résumons brièvement ci-dessous deux expériences de CoPréalisées au Québec, dans lesquelles les différentes clés de succès décrites précédemment ont été appliquées. L'une a été développée en présence auprès des superviseurs de stage en sciences de la santé et l'autre en recourant à un dispositif virtuel dans le cadre d'un programme de mentorat en médecine familiale.

Exemple 1 : Une CoP en présence auprès de superviseurs de stagiaires en sciences de la santé

Dans un centre de santé et de services sociaux regroupant plusieurs établissements de soins affiliés à l'Université Laval, Québec, l'idée d'une CoP composée de superviseurs de stagiaires de ces établissements a été initialement proposée en 2011 par un enseignant clinicien et formateur impliqué dans ce centre (LC). Lors d'activités de formation à la supervision qu'il animait, plusieurs participants exprimaient le besoin de réfléchir à des stratégies visant à optimiser l'exercice de leur rôle de modèle auprès des stagiaires. Ce besoin initial a conduit ce formateur à associer la direction de l'enseignement de ce centre dans l'élaboration, à titre de projet pilote, d'une CoP auprès de superviseurs et à y intégrer démarche évaluative de recherche-action.

Suite à un appel à participation, une $\mathrm{CoP}$ de dix superviseurs provenant de différentes professions de santé a vu le jour. Les participants visaient deux objectifs : (1) définir ce que signifie pour soi « être un clinicien enseignant modèle de rôle auprès des stagiaires »; (2) explorer et s'approprier des stratégies pédagogiques afin d'agir de manière intentionnelle et explicite en tant que modèle de rôle auprès d'eux. Lors de rencontres mensuelles tenues en présence pendant une année, les participants ont partagé leurs savoirs d'expérience et divers outils sur l'exercice du modèle de rôle en stage et en ont conçu un nouveau. L'expérience fut positivement évaluée autant par les participants que par leurs stagiaires qui avaient été questionnés sur l'exercice du modèle de rôle par leurs superviseurs. De nouvelles CoP furent réalisées avec quatre cohortes différentes de 2012 à 2016. Pour plus de détails, lire l'article correspondant à la référence [16].

Exemple 2: Une CoP virtuelle dans un programme de mentorat en médecine familiale

Le Québec fait face à des problèmes de recrutement de médecins de famille en clinique pour remplacer ceux qui s'acheminent vers la retraite. En 2015, le Collège québécois des médecins de famille a mandaté un groupe de travail pour explorer la possibilité du mentorat comme moyen de soutien de la relève en médecine de famille. Un programme formel de mentorat fut alors proposé à titre de projet pilote. D'une durée de seize mois, il visait à aider les médecins de famille en début de pratique à composer avec les enjeux et les défis inhérents à l'insertion professionnelle (par exemple: gestion du stress en début de pratique professionnelle, équilibre entre le travail et la vie personnelle, organisation de la pratique, identité professionnelle). Parmi les caractéristiques du programme initial, chaque professionnel mentoré choisit son mentor, puis identifie ses objectifs avec lui. Une communauté de pratique virtuelle vise à soutenir les mentors pour qui l'expérience du mentorat est souvent nouvelle. De même, des modalités technologiques facilitent la participation de mentorés et de mentors provenant de diverses régions géographiques du Québec. Finalement, une démarche de recherche-action de type participative vise à évaluer les impacts de ce programme.

Ce programme de mentorat en est à sa cinquième année d'existence. Année après année, il est très apprécié autant par les mentorés, leurs mentors, ainsi que par l'organisation. La CoP auprès des mentors est source d'apprentissage et de satisfaction aux dires des participants.

(Pour plus de détails, lire l'article correspondant à la référence [17] et consulter le site http://cqmf.qc.ca/soutiena-la-pratique/mentorat/). 


\section{Encadré 2. Des communautés de pratique en lien avec le contexte de crise sanitaire}

En lien avec le contexte actuel de crise sanitaire et des mesures de distanciation physique qu'elle impose, nous partageons nos réflexions au sujet des possibilités de mise en œuvre des communautés de pratique sous forme virtuelle ou hybride qui pourraient faire l'objet de quelques développements.

En lien avec le contexte de pandémie lié à la Covid-19, de nombreuses communautés de pratiques se sont improvisées. Elles visaient toutes un but commun, soit celui de s'adapter à un fonctionnement à distance, imposé par les règles de confinement en vigueur un peu partout. Ainsi, en un temps record, il est devenu possible pour des avocats de plaider en ligne, pour des médecins de suivre leurs patients à distance et pour des enseignants, qui n'y étaient pas habitués, d'enseigner à distance. Dans chacun de ces domaines, des communautés de pratique virtuelles ont été l'occasion pour les experts du «fonctionnement à distance» d'échanger avec des collègues qui devaient s'y habituer, avec ou sans enthousiasme. Bien que ces CoP improvisées n'aient pas été instaurées en cohérence avec toutes les clés de succès que nous présentons dans cet article, nous sommes d'avis qu'elles pourraient faire évoluer les pratiques. En effet, bon nombre de personnes qui étaient jadis réfractaires à l'intégration des technologies ont appris à s'en servir et elles ne pourront probablement plus s'en passer. Beaucoup ont réalisé que le fait d'œuvrer à distance permettait d'atténuer certaines contraintes de temps et de déplacements (déjà identifiées comme des obstacles au succès d'une $\mathrm{CoP}$ ), en plus de faciliter les collaborations internationales. Bien que certains aient utilisé les technologies, par dépit, en «attendant que ça passe », d'autres ont saisi des opportunités et ont appris à les exploiter pour transformer réellement les pratiques. Ces réelles transformations, que nous espérons durables, pourraient servir la qualité des CoP et en augmenter l'accessibilité. En effet, nous nous attendons à voir émerger des CoP hybrides qui profiteront des avantages de la présence et de ceux de la distance.

À ce sujet, un des auteurs (IS) s'implique dans un projet national de formation continue en santé maternelle et infantile au Burkina-Faso à l'intention de professionnels de la santé œuvrant dans ce domaine sur le terrain et provenant de diverses régions géographiques. L'implantation d'une CoP avec ces praticiens est en cours de planification. Ce «laboratoire-terrain» sera une autre occasion d'appliquer les clés de succès et d'en évaluer les impacts dans un contexte où plusieurs enjeux socio-culturels et organisationnels devront être pris en compte. Elle sera également l'occasion de mettre à l'essai une CoP hybride, qui combinera des échanges en présence, au Burkina, qui porteront sur les transferts dans la pratique et des échanges à distance qui seront l'occasion de réfléchir, avec des collègues à l'international et dans les diverses régions du pays, aux impacts de ces changements de pratique et à l'apport de la $\mathrm{CoP}$. Ce «laboratoire-terrain » sera aussi une occasion de réviser les clés de succès présentées, de façon de les adapter à l'«hybridisation» des pratiques.

\section{Références}

1. Davis DA, McMahon GT. Translating evidence into practice: Lessons for CPD. Med Teach 2018;40:892-5.

2. Légaré F, Freitas A, Thompson-Leduc P, Borduas F, Luconi $\mathrm{F}$, Boucher A, et al. The majority of accredited continuing professional development activities do not target clinical behavior change. Acad Med 2015;90:197-202.

3. Sargeant J, Wong BM, Campbell CM. CPD of the future: a partnership between quality improvement and competencybased education. Med Educ 2018;52:125-35.

4. Cruess RL, Cruess SR, Steinert Y. Medicine as a community of practice: Implications for medical education. Acad Med 2018;93:185-91.

5. McGrath C, Liljedahl M, Palmgren PJ. You say it, we say it, but how do we use it? Communities of practice: A critical analysis. Med Educ 2019;54:188-95.

6. Probst G, Borzillo S. Why communities of practice succeed and why they fail. Eur Manag J 2008;26:335-47.

7. Rashid A, Alexander K, Griffin A. New landscapes: Thinking beyond community in the conceptualisation of online medical education. Med Educ 2020;54:177-9.

8. Lessard A. Communauté de pratique. In: Jorro A, ed. Dictionnaire des concepts de la professionnalisation. Bruxelles : De Boeck Supérieur, 2013:57-60.
9. Charlier B. Apprentissage et communauté de pratique. In: Bourgeois É, Durand M (Dir.). Apprendre au travail. Paris : Presses Universitaires de France, 2012:99-110.

10. Li LC, Grimshaw JM, Nielsen C, Judd M, Coyte PC, Graham ID. Evolution of Wenger's concept of community of practice. Implement Sci 2009;4:11.

11. Collectif (sous la direction de Langelier L). Planifier sa communauté de pratique. In: Travailler, apprendre et collaborer en réseau. Québec: CEFRIO, 2005 [On-line] Disponible sur: https://numerique.banq.qc.ca/patri moine/details/52327/2006424.

12. Wenger E, McDermott R, Snyder WM. Seven principles for cultivating communities of practice. In: Cultivating Communities of Practice: a guide to managing knowledge. Boston: Harvard Business Press, 2002:1-9.

13. Arcand L. La communauté de pratique un outil pertinent: résumé des connaissances adaptées au contexte de la santé publique. Québec: Institut national de santé publique du Québec (INSPQ), 2018.

14. Wenger E, McDermott RA, Snyder W. Cultivating communities of practice: A guide to managing knowledge. Boston: Harvard Business Press, 2002.

15. de Carvalho-Filho MA, Tio RA, Steinert Y. Twelve tips for implementing a community of practice for faculty development. Med Teach 2019;42:1-7. 
16. Côté L, Perry G, Cloutier P-H. Développer son modèle de rôle en formation pratique : la contribution d'une communauté de pratique de cliniciens enseignants. Pédagogie Médicale 2013;14:241-53.

17. Côté L, Deschênes D, Hudon É, Galarneau S, Bolduc G. Le nouveau programme formel de mentorat du Collège québécois des médecins de famille. Can Fam Physician 2019;65:e475.

18. Bandura A. Social foundations of thought and action. Englewood Cliffs, New Jersey: Prentice-Hall, 1986.

19. Durning SJ, Artino AR. Situativity theory: a perspective on how participants and the environment can interact: AMEE Guide no. 52. Med Teach 2011;33:188-99.

20. Lave J, Wenger E. Situated learning: Legitimate peripheral participation. Cambridge: Cambridge University Press, 1991.

21. Wenger E. Communities of practice: Learning as a social system. Systems Thinker 1998;9:2-3.

22. Steinert Y. Faculty development: from rubies to oak. Med Teach 2020;42:429-35.

23. Wenger-Trayner E, Fenton-O'Creevy M, Hutchinson S, Kubiak C, Wenger-Trayner B, eds. Learning in landscapes of practice: Boundaries, identity, and knowledgeability in practice-based learning. London: Routledge, 2014.

24. Roberts J. Limits to communities of practice. J Manag Stud 2006;43:623-39.
25. Buckley H, Steinert Y, Regehr G, Nimmon L. When I say. Med Educ 2019;53:763-5.

26. Savard I, Bourdeau J, Paquette G. Considering cultural variables in the instructional design process: A knowledgebased advisor system. Computers \& Education 2020;145: 103722 .

27. Kotter J. Leading Change: Why Transformation Efforts Fail. Harvard Business Rev 2007;85:96-103.

28. Brien R. Science cognitive et formation. Québec : Presses de l'Université du Québec, 1990.

29. Kahlke RM, McConnell MM, Wisener KM, Eva KW. The disconnect between knowing and doing in health professions education and practice. Adv Health Sci Educ Theory Pract 2020;25:227-40.

30. Cook DA, Artino Jr AR. Motivation to learn: an overview of contemporary theories. Med Educ 2016;50: 997-1014.

31. Pemberton J, Mavin S, Stalker B. Scratching beneath the surface of communities of (mal) practice. Learn Organiz 2007;14:62-73.

32. Meagher-Stewart D, Solberg SM, Warner G, MacDonald J-A, McPherson C, Seaman P. Understanding the role of communities of practice in evidence-informed decision making in public health. Qual Health Res 2012;22: 723-39.

Citation de l'article : Savard I, Côté L. Optimiser le potentiel d'une communauté de pratique auprès de professionnels en santé : des clés de succès. Pédagogie Médicale 2021:22;43-52 